\title{
Capturing what matters to patients when they evaluate their hospital care
}

\section{R Fitzpatrick}

\section{Feedback from patients needs to be more specific if problems that can be targeted for change are to be identified.}

$\mathrm{t}$ is widely recognised that, when confronted by the increasingly common request to participate in a survey of their views, patients remain reluctant to express critical comments about the care they have received from hospital. The reasons for this reluctance are complex and include a desire not to appear ungrateful as well as recognition of inevitable limitations of health care. The results reported in this issue of Quality and Safety in Health Care by Jenkinson and colleagues $^{1}$ are further evidence of this phenomenon. One month after discharge from five hospitals in Scotland, patients received a questionnaire. Most of the $65 \%$ who completed and returned their questionnaire rated the quality of their care overall as either "very good" or "excellent", despite reporting on average 10 or five specific problems, respectively, out of a list of 40 possible problems.

One of the founding figures of the tradition of patient focused evaluation in the UK, Ann Cartwright, for many years demonstrated the advantages of using the methods of survey research to obtain patients' reports about specific experiences in their encounters with health care. ${ }^{2}$ Her work never depended on the more sophisticated methods of scaling and measurement of patients' views and attitudes associated with patient satisfaction research. The approach advocated here by the Picker Institute is firmly in Cartwright's tradition, focusing on the insights into shortcomings of health care obtained by surveys that ask about specific discrete experiences, rather than attitudes and evaluations.
Nevertheless, the study by Jenkinson et al illustrates the need to ask patients about both concrete experiences and their evaluations of those experiences Reading the report, hospital managers and clinicians might be tempted to focus their attention on far the most frequently reported specific problems reported by respondents-namely, their concerns about continuity of care. However, in multivariate analysis, problems classified as emotional support, respect for patient preferences, and physical comfort were stronger predictors of patients' overall evaluations of the quality of care. The results are strikingly similar to those obtained from virtually the same survey instrument completed by over 6000 patients discharged from 62 different hospitals in the US. ${ }^{3}$ The two studies together indicate that not all problems encountered by patients are equally important. Inclusion of questions about satisfaction or similar global judgements, combined with appropriate analyses, make it possible to identify the more important concerns. Questions about experiences and satisfaction may therefore both have a role.

Other research from the Picker Institute, using a carefully standardised instrument to assess patients' views about inpatient care in Germany, Sweden, Switzerland, UK and the US, suggests that there are many common features in the problems identified by patients across healthcare systems. ${ }^{4}$ By far the most frequently reported problems in all five countries were those relating to continuity of care between hospital and life after discharge. For all healthcare systems, fewest problems were reported regarding physical comfort. The use of standardised survey instruments across healthcare systems raises profoundly interesting questions about the determinants of quality in health care. Problems experienced by patients appear to be as much shaped by universal features of the culture and organisation of hospitals as by specific financial and administrative arrangements of individual healthcare systems.

Since the pioneering work of Cartwright and others, methodological issues have been increasingly recognised as important-whether the validity of instruments or potential biases from non-response to surveys. Sound methodology is an essential prerequisite for understanding the determinants of patients' evaluations of their health care and then for developing and testing interventions to improve the quality of care. It is also now clear that the more specific the focus of evaluative feedback from patients, the greater the likelihood that problems can be targeted for change. Results such as those reported here take us further along the route to improving health care as assessed by the recipient.

Qual Saf Health Care 2002;11:306

Correspondence to: Professor R Fitzpatrick, Professor of Public Health and Primary Care Division of Public Health and Primary Health Care, Institute of Health Sciences, University of Oxford, Oxford OX3 7LF, UK; raymond.fitzpatrick@nuffield.ox.ac.uk

\section{REFERENCES}

1 Jenkinson C, Coulter A, Bruster S, et al Patients' experiences and satisfaction with health care: results of a questionnaire study of specific aspects of care. Qual Saf Health Care 2002;11:335-9.

2 Cartwright A. Patients and their doctors. London: Routledge and Kegan Paul, 1967.

3 Cleary P, Edgman-Levitan S, McMullen W, et al. The relationship between reported problems and patient summary evaluations of hospital care. Qual Rev Bull 1992;18:53-9.

4 Coulter A, Cleary P. Patients' experiences with hospital care in five countries. Health Aff 2000;20:204-52. 